\title{
Structure and Patterns of Changtian-Style Costume of Miao Nationality
}

\author{
Wanchen Song* \\ Fuzhou University of International Studies and Trade, Fuzhou 350202, Fujian Province, China \\ *Corresponding author: Wanchen Song, 435792405@qq.com
}

\begin{abstract}
The emergence and development trend of the clothing worn by Miao people are closely related to their social lifestyles and folk customs. At the same time, the changes in the structures and patterns of their clothing have an indicative function to distinguish each branch, defining the range of age, marriage status, and so on. As a result, their clothing styles are diverse, and the patterns used for their clothing continuously evolve on the basis of earlier patterns.
\end{abstract}

Keywords: Miao costume; Changtian-style costume; Design pattern

Publication date: September 2021; Online publication: September 30, 2021

\section{Introduction}

The patterns on the clothing of Miao people carry the historical task of inheriting the national culture. Due to the limitation of cultural expression forms, the patterns, to a certain extent, have the functions of expressing, communicating, and reflecting the value identification of Miao women and their pursuit for a better life. Due to a long history, the words represented by these patterns and the specific meaning conveyed are hidden by a mysterious veil. As a carrier of the traditional national culture, their costumes reflect the material and spiritual life of the Miao nationality.

\section{Perfect combination of practical function and aesthetic function in Changtian-style costume}

Among all the ethnic costumes in China, Miao costumes have the most styles, the longest span of time, and the most gorgeous embroidery as well as silver decorations. In the early days, there are various exquisite embroidery techniques among the Miao people. At present, less and less people are able to master the traditional Miao embroidery techniques. The most common embroidery techniques now are counting yarn embroidery and needle embroidery. Changtian style is one of the representative styles using cross-stitch in the typical Miao costume.

The costume patterns with various themes by Miao people have been gradually formed and developed based on their national culture, lifestyles, and customs. They do not only reflect and express the Miao people's inner world of emotions, but also their understanding of nature as well as their yearning and prayer for a better life. These decorative patterns that have been formed along with the clothing and costumes of the Miao people are still used in daily clothing and articles with the characteristics of combining practical and aesthetic functions. The commonly used patterns are largely inspired by nature. They are created and expressed by freehand brushwork. For example, regular abstract geometric patterns with architecture, streams and mountains as design elements, and more intuitive floral patterns with rhododendrons and peonies express everything in the world. Although there was no concept of art at that time, people could only create according to their own cognition and aesthetic taste of nature. The selection and application of patterns are still irreplaceable in today's world. 


\section{Classic color of Changtian-style costume}

Miao costumes are usually in blue, black, white, and red. The colors of used in their embroidery patterns are usually natural pure colors, such as red, yellow, green, and white. The flowers are presented in block colors, showing a strong Miao costume style.

The environment they live in lays the foundation for the Miao people to upgrade their recognition of things from perceptual to rational. Miao women believe that the colors found in nature are the only color materials they need, and they should display them on their bodies to illustrate a kind of beauty originating from nature and surpassing nature. In regard to Changtian-style costume, natural colors and patterns, traditional hemp fabrics, simple and convenient styles, as well as delicate decorations from head to toe are the best interpretations of clothing culture.

\section{Structure and pattern of the Changtian-style costume of Miao nationality}

\subsection{Structure of the Changtian-style costume}

Changtian-style costume is the most typical clothing in Changtian Township, Buyi and Miao Autonomous County, Zhenning, Guizhou Province. Women dressed in Changtian-style would usually tie their hair in a bun on top of their head along with a green handkerchief with cross-stitch ribbon on their forehead. They would also wear pleated skirts made of batik and applique, and their collar, front, and sleeves will be decorated with cross-stitch. The end of the pleated skirt is made out of red batik cloth and decorated with a variety of traditional Miao patterns ${ }^{[5]}$. The front of the costume is a rectangular cross-stitch waist with a black base and traditional patterns of woven cross-stitch. The rear of the costume is tied with cross-stitch ribbons with colorful tassels under the ribbons. It is worth mentioning that the girdle of fixed ribbon is also very decorative, the width is relatively fine, the upper part is decorated with patterns, the length is enough to wrap four times around the waist, the legs are covered with white cloth or blue cloth leggings, and cloth shoes are used for footwear. Local women are excellent in craft work, such as cross-stitch and batik printing, which is reflected in their traditional costumes.

The most characteristic aspects of the Changtian-style costume are pleated skirts and caps, which are closely related to the lifestyles and historical origin of the Miao people. They also reflect the perfect combination of practical and aesthetic functions of clothing. The heavy headpiece, worn by Miao people by wrapping a cloth about 5 meters long around their heads, weighs about 4 kilograms. The outermost layer is a white-gray cloth decorated with traditional patterns. The hat is decorated with various tassels.

The pleated skirt in the costume is rectangular in shape; it is open and winds around the waist. The waist part is slightly longer than that of an ordinary dress; thus, it is not affected by the size of the body. The fabric of the pleated skirt is made from hemp material by traditional manual weaving. Hand sewing is first used to make the folds of the skirt. They are then fixed and left for a period of time to allow the pleats to come into shape. In addition, some would place the fixed pleated skirt in a steam pot with high temperature to finalize it. The appearance and craft of each branch skirt are generally the same. The differences are mainly reflected in the length, color, and pattern of the pleated skirt. In order to protect themselves from the cold weather, women's wear usually combines pleated skirts with leggings, while men's wear usually combines large shorts with leggings. Other than the beauty in the design of the pleated skirt, an important feature is that the wearer would not be hindered from carrying out activities. In the early days, in order to avoid wars, the inaccessible mountain was the habitat for Miao people, across the ditch and rivers without developed transportation. Therefore, walking was the main form of transportation in their daily lives. In view of that, the habit of walking fast came about and wearing pleated skirts did not affect them to take large steps when walking. Hence, pleated skirts were loved by women at that time and even at present times. 


\subsection{Pattern of the Changtian-style costume}

The pattern of Changtian-style costume emphasizes more on the shape and the rule in the design. The common organization forms include single pattern, continuous pattern, and suitable pattern. These organization forms are meticulous with many changes.

Single pattern is the most basic pattern organization form; its structure is relatively simple and selfcontained. This kind of pattern is mainly used in decorating cloth shoes. It is a balanced pattern alone without a fixed axis or center. The pattern emphasizes on natural beauty, but it is less used compared to the other two forms.

Continuous pattern can be divided into two-square continuous pattern and four-square continuous pattern. In the Changtian-style costume, the two-square continuous pattern is often used on the sleeves, collar, other parts of the jacket, as well as the decorative brocade belt ${ }^{[2]}$. This pattern is also common in blouses, mid-aprons, and millinery. The square continuous pattern is mainly used as a decoration in the Changtian-style costume, especially at the waist part to match and echo the role.

Suitable pattern refers to the pattern organization form suitable for a certain type of shape. There are various pattern forms, including symmetrical, independent balanced, left and right, flower edge, and closed. In addition, for silver ornaments and children's hats, suitable patterns are more common. The waist is an important part in Changtian-style clothing. The pattern used is a combination of continuous pattern and suitable pattern. It is a typical combination of different pattern organization forms for the same part.

From the perspective of pattern composition, although the pattern changes in density, compared to the composition of painting, it does not deliberately pursue a change in the master-follower relationship. Most of the patterns adopt the composition of flowers ${ }^{[4]}$. Even if decorative patterns appear in the form of repetition, isometric, symmetrical, cyclic-like, or as if being stacked, the pattern structure is meticulous, giving people a strong sense of order and rhythm with excellent decorative effect and visual impact.

\section{Origin and development of the patterns on Miao costume}

Animal patterns account for the largest number of existing patterns on clothing worn by Miao people. These patterns originate from the close relationship between the local Miao people and nature. Moreover, animals are used to express emotional sustenance ${ }^{[1]}$. The most representative animal pattern is the butterfly pattern, followed by the ox pattern, fish pattern, tiger pattern, frog pattern, and so on. The themes used in plant patterns are peony, maple leaf, camellia, cockscomb, and unknown flowers or plants found in the mountains or waters. Different patterns are shaped by concrete or abstract techniques of expression. There are also animal patterns and plant patterns that combine with other patterns which complement each other to set off a new pattern.

Geometric patterns are also highly generalized patterns designed by people through conscious abstract processing of objective things and graphics existing in nature ${ }^{[2]}$. The geometric patterns found on the Changtian-style costume are mainly triangle, diamond, and square. They are often used as decorations for the collar, hem, and other parts of the garment. The pattern is also reflected on the Changtian-style garment belt, which is the brocade belt.

After thousands of years of accumulation and innovation, the patterns on the clothing worn by Miao people have gradually changed into the current form. The themes, techniques, and meanings of the patterns have profound national elements, which cannot be created overnight. With the multicultural background and rapid information updates today, where foreign culture and modern thoughts have been undeniably accepted, there is a need to continuously inherit the spirit of national culture and development. 


\section{Conclusion}

The creation of a national costume is closely related to the living environment and cultural background. The Miao nationality is a nation with a long history and cultural background. Miao costumes are an important part of its cultural system. Their costumes and patterns reflect the development process and cultural deposition of the Miao people. They are also a record of the profound cultural essence, migration history, and living habits of the Miao people. The sublimation of their understanding and thoughts in the struggle with nature form their unique national costume and folk culture. Each style of clothing reflects the Miao people's yearning and pursuit for a better life.

\section{Disclosure statement}

The author declares that there is no conflict of interest.

\section{References}

[1] Chen L, 2012, Research on The Composition form of Miao Costume Patterns. Kunming University of Science and Technology.

[2] Weng C, 2014, Talk about Miao Embroidery. Silk.

[3] You Y, 2010, Research on The Pattern and Graphic Elements form Composition of Miao Nationality's Costumes in Yunnan. Yunnan Arts University.

[4] Lu G, Ma Y, 2011, On the Inheritance and Innovation of Cross-Stitch Technology. Shandong Textile Economy.

[5] Wu S, 2000, Chinese Miao Costume Chart. Guizhou People’s Publishing House. 